\title{
Peptide Deformylase (def) is essential in Mycobacterium smegmatis, but the essentiality is compensated by inactivation of methionine formylation
}

\author{
Noga Naor ${ }^{\dagger}$, Omer Gadot ${ }^{\dagger}$, Michal Meir $^{2}$ and Daniel Barkan ${ }^{1 *}$ (D)
}

\begin{abstract}
Background: Co-translational processes in bacteria are attractive drug targets, but while some processes are essential, others are not. The essentiality of Peptide Deformylase (PDF, def) for vitality of mycobacteria was speculated, but never unequivocally proven.

Results: Here we show by targeted deletion experiments that def can only be deleted from M. smegmatis when an additional copy is present; that prior deletion of tRNA ${ }^{\text {fMet }}$-Formyl Transferase (FMT, encoded by fmt) renders def completely dispensable; and that re-introduction of fmt into a $\Delta$ def mutant is not possible - constituting a definitive proof for the essentiality of def in mycobacteria.

Conclusions: Peptide deformylase is essential in M. smegmatis, but the fact that inactivation of fmt renders the gene completely dispensable, and thus any inhibitor of def useless, casts doubt on the usefulness of PDF as a drug-target in mycobacteria.
\end{abstract}

Keywords: Mycobacteria, Protein synthesis, Co-translation, Peptide Deformylase, Formyl Transferase

\section{Background}

Mycobacteria continue to be a major health threat throughout the world. M. tuberculosis causes over 1.6 million deaths worldwide [1], but the burden of $M$. leprae and M. ulcerans (mostly in the developing world), and that of pathogenic rapidly-growing mycobacteria such as $M$. abscessus and M. kansasii is rising as well [2-4], mostly in the developed world. Many mycobacteria exhibit substantial drug resistance and tolerance, making the need for novel therapeutics more urgent [5]. Understanding of physiologic processes unique to bacteria is important for identifying essential pathways that could be targeted. One such process is the prokaryotespecific formylation and de-formylation of the methionine that starts the synthesis of every protein $[6,7]$. In

\footnotetext{
* Correspondence: daniel.barkan@mail.huji.ac.il.

${ }^{\dagger}$ Noga Naor and Omer Gadot contributed equally to this work.

${ }^{1}$ Koret School of Veterinary Medicine, The Robert H. Smith Faculty of

Agriculture, Food and Environment, The Hebrew University of Jerusalem

Rehovot Campus, Rehovot, Israel

Full list of author information is available at the end of the article
}

mycobacteria, methionine is first formylated by tRNA ${ }^{\mathrm{f}-}$

Met-Formyl Transferase (FMT, encoded by fmt), and only a formylated methionine can be used as the first amino acid in a new protein $[8,9]$. Shortly after it emerges from the ribosome, the leading methionine is de-formylated by Peptide Deformylase (PDF, encoded by def). Following deformylation, methionine is removed from the majority of mycobacterial proteins by either Methionine Amino-Peptidase A (MetAPa, encoded by mapA) or MetAPc (encoded by mapB) $[8,10]$. FMT was suggested to be essential by a transposone-mutant library analysis in M. tuberculosis [11-13], but directed deletion experiments showed it to be completely dispensable for $M$. smegmatis, and to cause only mild growth retardation in $M$. bovis when deleted [9]. In MetAP's, chemical inhibition studies first suggested mapA was essential whereas $m a p B$ was not [8], but directed gene deletion experiments on $M$. bovis proved it to be the other way around - mapB, and not mapA, was the essential one, with some redundancy [10]. 
PDF (and $d e f$ ) is postulated to be vital in all bacteria probably as MetAP's cannot function on proteins where the formyl group was not removed [14]. def was suggested to be essential in $M$. bovis-BCG, and a chemical inhibitor of it had antibacterial activity [15]. However, given the fact chemical inhibition experiments, as well as the genetic method used to prove essentiality in that study, may mistake a slow-growth phenotype for a lethal one, thus leading to incorrect essentiality attributions (as was the case for fint and mapA/mapB). We opted to definitively examine PDF's role in mycobacterial viability by deletion experiments, as well as deletion on an fmtnull background.

\section{Results}

def cannot be deleted from the chromosome of $M$. smegmatis

For the deletion of $d e f$, we used the two step allelic exchange system, for which we constructed the plasmid pDB354. The plasmid contains both zeocin ${ }^{R}$ and strepto$\operatorname{mycin}^{R}$ resistance genes (for positive selection), $s a c B$ (for negative selection), and the two flanking regions of def ( $~ 600$ bp each, placed adjacent to each other). The plasmid was electroporated into wt $M$. smegmatis, colonies were selected on zeocin and streptomycin, and examined for successful recombination at one of the def flanking regions by PCR. One correct colony was found, and named M.smeg ${ }^{i n t}$ (for "intermediate"). These bacteria were grown without antibiotic selection for 15 generations to allow for a second recombination event, and plated on sucrose $10 \%$ for negative selection. One hundred colonies that grew on sucrose were patched on either no selection or zeocin/streptomycin to differentiate true second recombination events from $s a c B$ inactivating mutations. Sixty colonies were found to be true second recombination events (sucrose $\mathrm{e}^{\mathrm{R}} /$ Zeocin $^{\mathrm{S}} /$ streptomycin $^{\mathrm{S}}$ ). All were examined by PCR that produces an $1800 \mathrm{bp}$ fragment in the case of reversal to wt, and $1200 \mathrm{bp}$ in case of a def deletion genotype. All 60 colonies were found to revert to wt, with none having the deletion genotype (Fig. 1). We therefore conclude that deletion of def is, at the very least, highly deleterious in wt $M$. smegmatis.

In the presence of an additional copy, def can be deleted We complemented M.smeg ${ }^{\text {int }}$ with an additional copy of $d e f$ at the $a t t B$ site, on a hygromycin-selected plasmid. We then proceeded to the second recombination as described in the previous section. This time, out of the 12 colonies that underwent a second recombination event, four were def deletions, whereas the other eight reverted to wt genotype (Fig. 2), indicating the in the presence of an additional copy of $d e f$, the native def is completely dispensable (and that the deletion strategy used was valid).

The lethality of def deletion is compensated by tRNA ${ }^{\text {fMet }}$ Formyl Transferase (FMT, fmt) inactivation

The physiologic function of the def product (Peptide Deformylase, PDF) is to remove the formyl group from the leading methionine. Since this formyl group is originally attached by another enzyme, the tRNA ${ }^{\text {fMet }}$-Formyl Transferase (FMT, encoded by $f m t$ ), we speculated that on an fint-deletion background, def may become dispensable. We previously described a complete deletion of fmt in M. smegmatis (strain $\mathrm{mDB} 22$ ) and in $M$. bovis [9]. Contrary to previous reports, the deletion of fmt was not lethal, causing only a trivial growth defect in M. smegmatis, and a 2-3 fold slower growth in $M$. bovis [9]. We decided to examine if fmt deletion renders def completely dispensable. We created an $\mathrm{mDB} 22^{\text {int }}$ strain

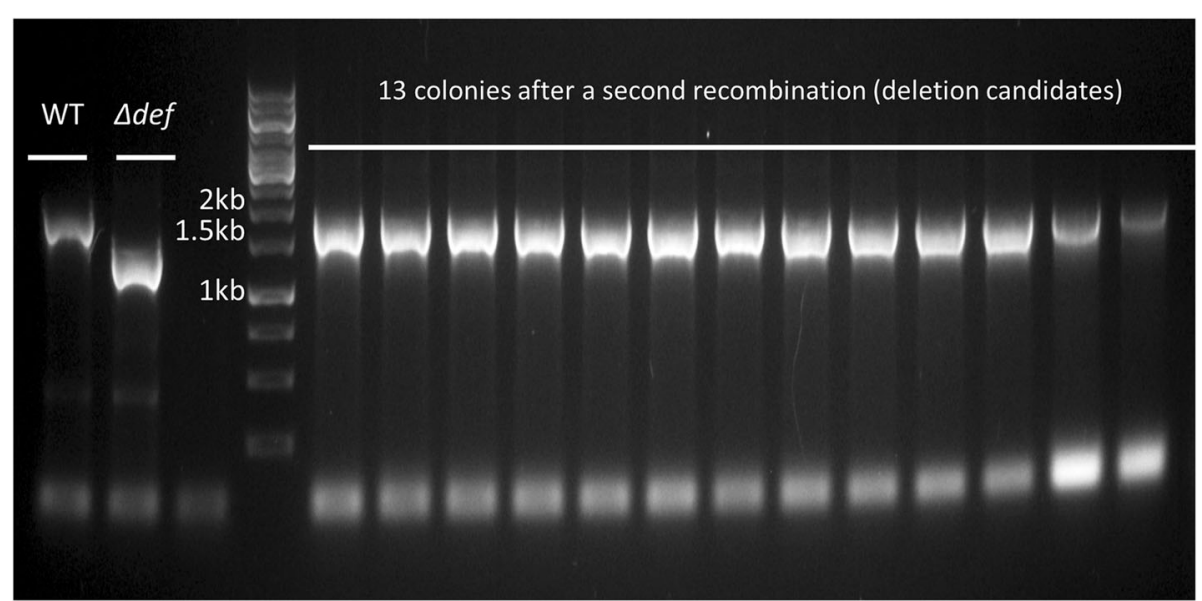

Fig. 1 def cannot be deleted from wt M. smegmatis. On the right, colonies after a confirmed second recombination event were checked by PCR to differentiate def-deletion (resulting in $1.2 \mathrm{~kb}$ product) from reversal to wt (1.8 kb fragment). 13 colonies are shown, out of 60 examined. On the left - wt control, and a true $\Delta$ def mutant (obtained as described in section 3.2) 


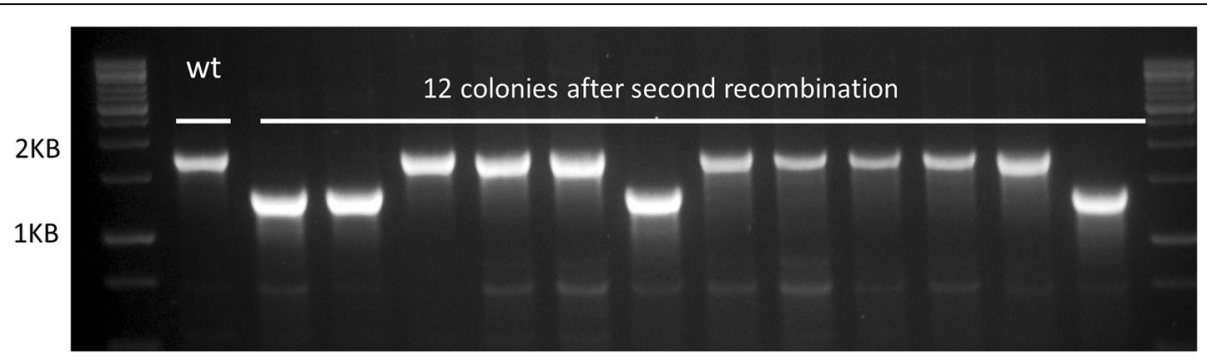

Fig. 2 With pre-complementation with an additional copy of def, a deletion of the native gene is easily obtainable. Right - 12 colonies after second recombination. 4 of them have the PCR product obtained from a $\Delta$ def genotype (1200 Bp). Left - wt control (1800 bp)

using pDB354 (as was done for def deletion on wt background), proceeded to second recombination, and then examined colonies using the previously described PCR. This time we easily obtained a deletion mutant, with 5 colonies out of 10 candidates being def knock-outs (Fig. 3). This confirms that in the absence of fmt, def is completely dispensable. One of these colonies was arbitrarily chosen for continued work, and named mDB224 (M. smegmatis $\Delta$ def/ $\Delta f m t)$.

\section{fmt is incompatible with a $\Delta d e f / \Delta f m t$ genotype}

The fact def could only be deleted from $M$. smegmatis (and M. bovis, previously) when an additional copy was present at the attB site, does not constitute a proof it is completely essential - as a viable, but with a substantial growth defect mutant, would not be found in these types of experiments. We therefore wanted to examine if fmt could be re-introduced into a double def/fmt null mutant. If def is completely essential (on an fmt-positive background), then this re-introduction would yield no colonies. Slow-growing mutants will be discovered, as there are no normal-growing bacteria to mask them. We therefore prepared two genetic constructs to reintroduce $f m t$ into the $\mathrm{fm} t / \mathrm{def}$ double deletion mutant (mDB224): fmt smegmatis $(\mathrm{pDB} 384)$ or fmt tuberculosis (pDB332) on a multi-copy episomal vector. Both plasmids had a stringent double selection (zeocin and kanamycin) to prevent background colonies. All electroporations were done with an empty vector control (also expressing mCherry, with resulting pink colonies). Whereas the control yielded multiple colonies, the fmtcontaining plasmids yielded none (Fig. 4). Insertion of both pDB332 and pDB384 into wt $M$. smegmatis yielded multiple colonies (data not presented), showing the plasmids themselves are transformable, and confer antibiotic resistance as expected. We therefore conclude that fmt re-introduction is incompatible with a def-deletion background, providing unequivocal proof of def essentiality for viability, and not just for normal or near-normal growth.

\section{Discussion}

Protein synthesis and co-translational processes are an attractive target for development of antibacterials, as there are several steps quite unique to prokaryotes specifically the formylation and de-formylation of the leading methionine [6, 7]. However, targeted enzymes should be those shown to be essential for viability, and the essentiality is sometimes difficult to prove. Fmt was previously claimed to be essential, based on transposon-mutant library analyses [11-13]. However, these analyses are strongly biased against viable but slow-growing mutants, and directed deletion experiments in $M$. smegmatis and in M. bovis unequivocally

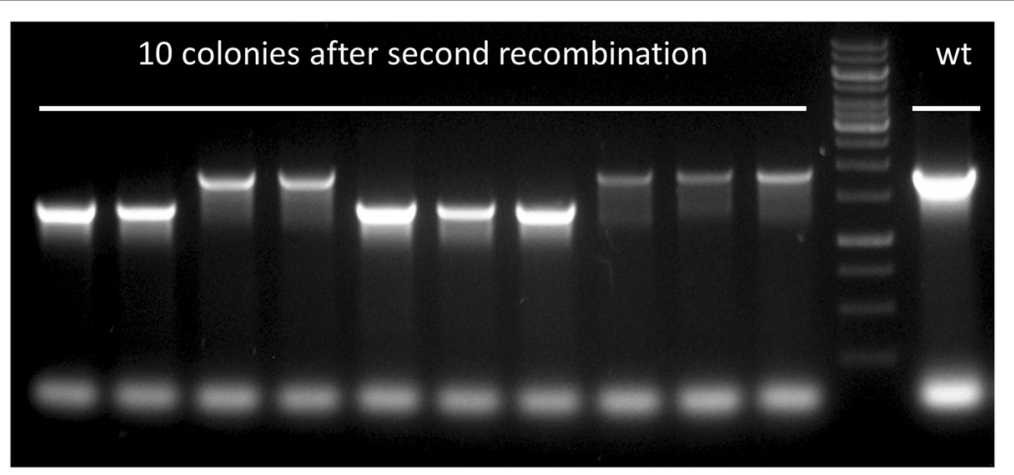

Fig. 3 On a $\Delta f m t$ background, deletion of def is highly efficient. Left: 10 candidate colonies. Right: wt control. The leftmost colony was later re-named mDB224 


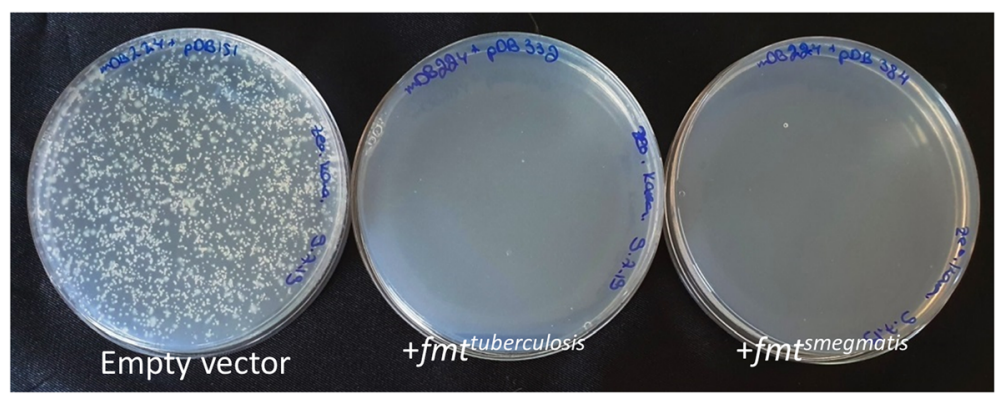

Fig. 4 A functional $f m t$ is incompatible with a $\Delta f m t / \Delta$ def genotype. mDB224 ( $\Delta f m t / \Delta$ def) was electroporated with a control vector (left), control+fmt $t^{\text {tuberculosis }}$ (middle), and control+fmt $t^{\text {smegmatis }}$ (right). The picture was taken after 4 days incubation, but plates were kept for 10 days before discarding, not to miss slow-growing mutant

showed the gene was dispensable [9]. Similarly, a chemical inhibition assay suggested MetAP1a was essential in M. tuberculosis, and that MetAP1c was not [8]. Again, directed deletion experiments showed the opposite [10]. As opposed to the well-established, complete essentiality of MetAp's in all life forms [17, 18], the role of DEF in different organisms is deemed controversial [19]. A targeted deletion in M. bovis$B C G$ only yielded colonies when an additional copy of the gene was present (in a two-step allelic exchange technique), leading to the conclusion def was essential (as also expected from what is known in other bacteria) [15]. However, these analyses are based on testing of several dozen colonies appearing on a negative-selection plate. Even if the gene in question is completely dispensable, at least $50 \%$ of colonies will revert to wt, and if the deletion of the gene is viable but causes growth retardation, the proportion of the deletion mutants will become much smaller, as the wt colonies will appear well before the deletion ones. Therefore, this kind of experiment can suggest an important role in growth, but not absolute essentiality. Here, we first repeated the previous path, where we showed the gene could be deleted when an additional copy was present, and no deletion mutants could be obtained when no pre-complementation was done. We then showed that on a background of fmt deletion, the role of def becomes completely insignificant, as evident by a $50 \%$ deletion rate versus reversal to wt. However, we then showed that re-introduction of $f m t$ (both smegmatis and tuberculosis origin) is impossible into a mutant that has no def - meaning a functional $f m t$ is incompatible with a $\Delta d e f$ genotype - and this constitutes a definitive proof of def essentiality.

Why deformylation is essential is not completely clear. One can postulate the formyl group blocks the activity of the methionine aminopeptidase enzymes (MetAP), who's activity is known to be essential in all life forms [17], including prokaryotes. One should remember the leading methionine is not removed from all synthesized proteins, and it is possible that in proteins retaining this methionine, the formyl group may be retained as well, with no compromise of viability. This may explain how mitochondrial proteins in eukaryotes constantly retain the formyl group [18, 20, 21]. Nonetheless, genes homologous to def were shown to exist in the nuclear genomes of higher eukaryotes, together with studies demonstrating mitochondrial DEF expression with highly selective functions in human cells [19, 22, 23]. This suggests DEF does play a role in specific deformylations in eukaryotic organisms as well. Moreover, it was shown that PDF of human origin could compensate for def deficiency in an E. coli mutant with conditional expression of def [24], pointing the conjecture that functionally, human PDF might be similar to the bacterial one.

The essentiality of $d e f$ raises the question of whether it can serve as an effective drug target. A previous study showed chemical inhibition of def does have antimycobacterial properties [25]. However, it appears from our study that in the case of fmt inactivation, the inhibition of def becomes irrelevant to growth. The barrier for spontaneous resistance mutations is therefore relatively low, as inactivating mutations (unlike mutations that retain activity, but do not bind an inhibitor) are fairly common. It remains to be seen if the co-translation pathway is indeed a viable drug target.

\section{Conclusions}

PDF (def) was unequivocally shown to be essential in $M$. smegmatis, and probably in all mycobacteria. However, as genetic inactivation of FMT $(f m t)$ rendered the bacteria completely neutral as for the existence or absence of PDF, the role of PDF as a viable drug target remains questionable. It could, however, be part of a combination regimen, with other drugs that act on non-related targets.

\section{Methods}

Strains and growth conditions

Mycobacterium smegmatis $\left(m c^{2}-155\right)$ were grown in Middlebrook 7H9 media, supplemented with $0.5 \%$ glycerol, 
$0.5 \%$ dextrose, and $0.02 \%$ Tween 80 . For solid agar plates, we used 7H10 Middlebrook with glycerol and dextrose, but no tween. Antibiotic concentrations were: kanamycin $40 \mu \mathrm{g} / \mathrm{mL}$, zeocin $25 \mu \mathrm{g} / \mathrm{mL}$, streptomycin $20 \mu \mathrm{g} / \mathrm{mL}$, and hygromycin $50 \mu \mathrm{g} / \mathrm{mL}(150 \mu \mathrm{g} / \mathrm{mL}$ for E. coli). $\Delta f m t$ M. smegmatis was previously described [9].

\section{Deletion and complementation of def}

Deletion was done in the two-step allelic exchange, as previously widely described [16]. The plasmid used for the first step recombination was pDB354. The upstream and downstream flanking regions of def were PCR amplified from $M$. smegmatis genome, fused into a single PCR product using primers defsmko1-5, and cloned into a plasmid containing zeocin ${ }^{R}$, streptomycin ${ }^{R}$ and $s a c B$. After the second recombination, to differentiate between a deletion mutant and reversal to wt, we used primers defKOchqD and defKOchqU that produce an $1800 \mathrm{bp}$ fragment in wt, and $1200 \mathrm{bp}$ fragment in a deletion mutant. To verify the 1200 bp product is indeed the result of a successful deletion, the PCR product was sent for Sanger sequencing, and was shown to be identical to the wt-genotype, $1800 \mathrm{bp}$ product at both ends, but to lack the bulk of the def gene, as constructed in the plasmid used for the deletion (pDB354), resulting in shortening by $\sim 600 \mathrm{bp}$. To complement with an additional

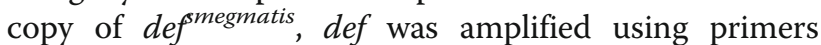
defSmU and defSmD, and cloned into the integrating plasmid pYUB412. All the primers sequences are shown in Additional file 1: Table S1.

\section{Complementation with $\mathrm{fmt}$}

A multicopy episomal plasmid, selected by zeocin and kanamycin, and also expressing mCherry, with a copy of fmt $t^{\text {tuberculosis }}$ was previously described by us (pDB332, 9]. A similar plasmid (with no mCherry) for $f m t^{\text {smegmatis }}$ was constructed using primers fmt smg_R_xbaI, and fmt smg_BamHI, and cloned into pDB151, producing pDB384. Electroporations were done as previously widely described.

\section{Supplementary information}

Supplementary information accompanies this paper at https://doi.org/10. 1186/s12866-019-1611-7.

Additional file 1: Table S1. Primers (oligonucleotides) used in this study.

\section{Abbreviations}

FMT: Formyl methionine transferase; MetAP: Methionine aminopeptidase; PDF: Peptide deformylase; WT: Wild type

Acknowledgements

Not applicable.

\section{Author's contributions}

$\mathrm{NN}$ and $\mathrm{OG}$ performed most of the experiments. NN and MM participated in the draft and final writing. MM and DB conceptualized the study. DB performed experiments and wrote the final version.

\section{Funding}

MM is supported by an internal grant from Rambam Medical Canter, "Ofakim", and by the Israeli Science Fund (ISF). The granting body did not participate in the design of the study, in collection, analysis, and interpretation of data, nor in writing the manuscript.

Availability of data and materials

No large databases were used for this study. The datasets used and/or analyzed during the current study are available from the corresponding author on reasonable request.

Ethics approval and consent to participate

Not applicable.

Consent for publication

Not applicable.

\section{Competing interests}

The authors declare that they have no competing interests.

\section{Author details}

${ }^{1}$ Koret School of Veterinary Medicine, The Robert H. Smith Faculty of Agriculture, Food and Environment, The Hebrew University of Jerusalem Rehovot Campus, Rehovot, Israel. ${ }^{2}$ The Ruth Rappaport Children's Hospital, Rambam Health Care Campus, Haifa, Israel.

Received: 20 August 2019 Accepted: 6 October 2019

Published online: 26 October 2019

\section{References}

1. WHO. Global Tuberculosis Report 2017. Geneva, Switzerland; 2018.

2. De Groote MA, Huitt G. Infections due to rapidly growing mycobacteria. Clin Infect Dis. 2006;42:1756-63.

3. Snapper SB, Melton RE, Mustafa S, Kieser T, Jr WRJ. Isolation and characterization of efficient plasmid transformation mutants of Mycobacterium smegmatis. Mol Microbiol. 1990;4:1911-9.

4. Duker AA, Portaels F, Hale M. Pathways of Mycobacterium ulcerans infection: a review. Environ Int. 2006:32:567-73.

5. Zhang $Y$. The magic bullets and tuberculosis drug targets. Annu Rev Pharmacol Toxicol. 2005:45:529-64.

6. Anderson WF. Initiation of protein synthesis in prokaryotic and eukaryotic systems summary of EMBO workshop held in Noordwijkerhout, the Netherlands on 17-19 April 1974. FEBS Lett. 1974;48:1-6.

7. Giglione C, Pierre M, Meinnel T. Peptide deformylase as a target for new generation, broad spectrum antimicrobial agents: MicroReview. Mol Microbiol. 2000:36:1197-205.

8. Olaleye O, Raghunand TR, Bhat S, He J, Tyagi S, Lamichhane G, Gu P, Zhou J, Zhang Y, Grosset J. Methionine aminopeptidases from Mycobacterium tuberculosis as novel antimycobacterial targets. Chem Biol. 2010;17:86-97.

9. Vanunu M, Lang Z, Barkan D. The gene fmt, encoding tRNA fMet-formyl transferase, is essential for normal growth of $M$. bovis, but not for viability. Sci. Rep. 2017;7:15161

10. Vanunu M, Schall P, Reingewertz T-H, Chakraborti PK, Grimm B, Barkan D. MapB protein is the essential methionine Aminopeptidase in Mycobacterium tuberculosis. Cells. 2019;8:393.

11. Griffin JE, Gawronski JD, DeJesus MA, loerger TR, Akerley BJ, Sassetti CM. High-resolution phenotypic profiling defines genes essential for mycobacterial growth and cholesterol catabolism. PLoS Pathog. 2011;7: e1002251.

12. Lamichhane G, Zignol M, Blades NJ, Geiman DE, Dougherty A, Grosset J, Broman KW, Bishai WR. A postgenomic method for predicting essential genes at subsaturation levels of mutagenesis: application to Mycobacterium tuberculosis. Proc Natl Acad Sci. 2003;100:7213-8.

13. Sassetti CM, Boyd DH, Rubin EJ. Genes required for mycobacterial growth defined by high density mutagenesis. Mol Microbiol. 2003;48:77-84. 
14. Yuan Z, Trias J, White RJ. Deformylase as a novel antibacterial target. Drug Discov Today. 2001;6:954-61.

15. Teo JWP, Thayalan P, Beer D, Yap ASL, Nanjundappa M, Ngew X, Duraiswamy J, Liung S, Dartois V, Schreiber M. Peptide deformylase inhibitors as potent antimycobacterial agents. Antimicrob Agents Chemother. 2006:50:3665-73.

16. Pavelka MS, Jacobs WR. Comparison of the construction of unmarked deletion mutations in Mycobacterium smegmatis, Mycobacterium bovis bacillus Calmette-Guerin, and Mycobacterium tuberculosis H37Rv by allelic exchange. J Bacteriol. 1999;181:4780-9.

17. Bradshaw RA, Brickey WW, Walker KW. N-terminal processing: the methionine aminopeptidase and $\mathrm{Na}$-acetyl transferase families. Trends Biochem Sci. 1998;23:263-7.

18. Meinnel T. Peptide deformylase of eukaryotic protists: a target for new antiparasitic agents? Parasitol Today. 2000;16:165-8.

19. Lee MD, She Y, Soskis MJ, Borella CP, Gardner JR, Hayes PA, Dy BM, Heaney ML, Philips MR, Bornmann WG. Human mitochondrial peptide deformylase, a new anticancer target of actinonin-based antibiotics. J Clin Invest. 2004; 114:1107-16.

20. Walker JE, Lutter R, Dupuis A, Runswick MJ. Identification of the subunits of F1FO-ATPase from bovine heart mitochondria. Biochemistry. 1991;30:536978.

21. Yagi T, Hatefi Y. Identification of the dicyclohexylcarbodiimide-binding subunit of NADH-ubiquinone oxidoreductase (complex I). J Biol Chem. 1988:263:16150-5

22. Nguyen KT, Hu X, Colton C, Chakrabarti R, Zhu MX, Pei D. Characterization of a human peptide deformylase: implications for antibacterial drug design. Biochemistry. 2003:42:9952-8.

23. Serero A, Giglione C, Sardini A, Martinez-Sanz J, Meinnel T. An unusual peptide deformylase features in the human mitochondrial $\mathrm{N}$-terminal methionine excision pathway. J Biol Chem. 2003;278:52953-63.

24. Leeds JA, Dean CR. Peptide deformylase as an antibacterial target: a critical assessment. Curr Opin Pharmacol. 2006;6:445-52.

25. Apfel CM, Locher H, Evers S, Takács B, Hubschwerlen C, Pirson W, Page MGP, Keck W. Peptide deformylase as an antibacterial drug target: target validation and resistance development. Antimicrob Agents Chemother. 2001;45:1058-64.

\section{Publisher's Note}

Springer Nature remains neutral with regard to jurisdictional claims in published maps and institutional affiliations.

Ready to submit your research? Choose BMC and benefit from:

- fast, convenient online submission

- thorough peer review by experienced researchers in your field

- rapid publication on acceptance

- support for research data, including large and complex data types

- gold Open Access which fosters wider collaboration and increased citations

- maximum visibility for your research: over $100 \mathrm{M}$ website views per year

At $\mathrm{BMC}$, research is always in progress.

Learn more biomedcentral.com/submissions 